\title{
7. COMMISSION DE LA MECANIQUE CELESTE
}

Président: M. G. M. Clemence.

Membres: MM. Armellini, Belorizky, Brouwer, Bucerius†, Chazy†, Chebotarev, Cox, Cunningham, Duboshin, Eckert, Fabre, Hagihara, Hamid, Heinrich, Herget, Herrick, Jeffreys, Lemaître, Milankovitch, Mineur $\dagger$, Moiseiev†, Sadler, Sconzo, Slavenas, Stumpff, Subbotin, van Woerkom, von Zeipel, Wilkens, Zagar.

Recommendation by M. Herrick:

The Commission recommends that accurate observations of Icarus be made in anticipation of its close approach in 1968 by astronomers equipped with photographic equipment that will reach the necessary magnitude. It expresses its appreciation for the observations made under difficult circumstances at the Radcliffe and Cape Observatories.

We report with regret the death of our esteemed members Henri Mineur, on 7 May I954 and Jean Chazy, on 9 March 1955. A necrology of the former by Daniel Barbier has appeared in Annales d'Astrophysique and of the latter by André Danjon in Comptes Rendus.

\section{Progress of Research}

The following report should be supplemented by reference to the reports of Commissions $4,17,20,22,28,3 I$, and 33 , all of which fall to some extent in the field of celestial mechanics.

\section{Rotation of the Earth}

The annual variation in the speed of rotation has been observed at several observatories, with similar but not identical results. In these studies the standard of reference is furnished by vibrating quartz crystals, and the observed discordances are presumably due to differences in the performance of the crystals. Further details will be found in the report of Commission 3I. Mintz and Munk have shown that most of the variation can be accounted for by the action of winds and tides.

Van den Dungen, Cox, and van Mieghem have studied the effect of meridianal winds on the motion of the pole.

Brouwer has shown that the irregular fluctuations in the speed of rotation are of a kind that would be produced by small random changes in acceleration; the associated changes in speed are not abrupt but gradual. The cause remains unknown, but it has been suggested that turbulence in the core, with electromagnetic coupling of the mantle, may account for it. In any case the secular retardation of the speed of rotation, as determined by observations of the Sun and Moon, must now be recognized to be much more uncertain than was formerly supposed. Holmberg has even suggested that resonance between the natural period of the atmosphere and the period of the solar tide may counteract tidal friction, thus maintaining the average speed of rotation constant over a very long time; this hypothesis is not contradicted by observations.

Sekiguchi has studied the effects of viscosity of the core and finds limits between which a phase-shift of the nutation can occur. He has also studied the effect of ocean tides of short period on the rotation of a yielding earth.

\section{Problem of three bodies}

Pariysky and Hilmi have confirmed the possibility of capture announced by Schmidt in 1943. Pariysky has shown numerically how a binary star can result from an encounter of three stars of equal mass moving in the same plane. Merman has determined the conditions that make the capture possible, and has thus been able to answer some important questions posed by Chazy.

Radziewsky has studied the effect of radiation-pressure in the problems of two bodies and three bodies, also the motion in a pressure-cloud of constant density. 
Stumpff has attempted to solve the restricted problem by the use of a rigorous algebraic differential equation of the third order, but finds complications.

Bucerius has derived and tested a method for orbit determination by the method of Gauss, simultaneously taking into account the third body by the use of integral equations.

Miyahara has published a study of non-contact canonical transformations.

Wilkens has made a general study of the invariant integrals of the problem, with special reference to the integral of Poincaré.

Belorizky has shown that Sundman's variable for the regularization of the problem does not in fact regularize it over the entire plane of the variable.

Lemaître has published some contributions.

\section{Planetary theory}

Wilkens has studied the 18:5 term in the motion of Encke's comet disturbed by Jupiter.

Sconzo has studied the numerical integration of orbits using algebraic methods with a large step-interval.

Kozai has applied a modification of Woltjer's method for the Hyperion-Titan system to the minor planet Thule. After obtaining the terms depending on the libration-argument he gets the synodic terms by semi-analytic numerical integration.

Bagenov has studied problems relative to the estimation of error in series-developments of the disturbing function.

Sharaff has calculated analytically the first-order perturbations of Pluto and has accomplished an important part of the work for the second-order perturbations. Preparatory to a comparison with observations, Lavdovsky has made a systematic study of the observations taken with the astrograph at Pulkovo I930-5I.

Subbotin reports that the first-order perturbations of Ceres by all the large planets have been calculated and published; the calculation of the second-order perturtiations is in progress.

Musen has published a general study of the method of the variation of parameters.

Van Woerkom has calculated new and extensive tables for the development of the disturbing function, shortly to be published; they include tabulations of the logarithmic derivatives of the Laplace coefficients.

Brouwer and van Woerkom have undertaken preliminary work on general theories of Uranus, Neptune, and Pluto, using Brouwer's method in rectangular coordinates.

Woolard has made a systematic study of pre-discovery observations of Uranus, and compared them with the coordinates obtained in the numerical integrations for the five outer planets; the results have been used by Brouwer in a new orbit-correction, shortly to be published.

Brouwer and Clemence, using the coordinates of the five outer planets obtained by numerical integration for 407 years, have evaluated the area-integrals and the energyintegral for five dates with high precision; the results, to be published shortly, fully confirm the accuracy of the numerical integrations.

Clemence has calculated the second-order perturbations of Mars by Venus, Earth, Jupiter, and Saturn, including all first powers, squares, and products of the disturbing masses wherever they produce appreciable effects; a comparison with observations is in progress.

\section{Attraction between bodies of finite size}

A. A. Orlov has studied the quasi-periodic motion of a point-mass under the attraction of a spheroid, and the problem of the integration of the equations of disturbed motion by the method of a very small parameter in rectangular co-ordinates.

H. R. Morgan and Sekiguchi have independently shown that the coefficients of the terms of the nutation having half the period of the Moon's mean longitude are correctly given by the theory for a rigid Earth, within the errors of observation. 
Woolard has redeveloped the theory of precession and nutation for a rigid Earth, obtaining series-expressions with numerical coefficients of very high precision; the work is based on Newcomb's theory of the motion of the Earth and Brown's theory of the motion of the Moon.

\section{Elliptic motion}

Belorizky has proposed a new method for the calculation of the eccentric anomaly in nearly parabolic orbits by means of series that converge more rapidly than the classical ones.

The development of new observational techniques by Whipple now permits the calculation of orbits or meteors with precision comparable to preliminary orbits of comets.

\section{G. M. Clemence President of the Commission}

Compte rendu de la séance. 30 août I955

Président: Dr G. M. Clemence.

SecrétaIRE: Prof. M. Subbotin.

Les membres de la commission approuvent le rapport préliminaire sans modification.

On passe ensuite à la discussion de la recommandation de M. Herrick. La recommandation de $\mathrm{M}$. Herrick est approuvée, et la commission charge le secrétaire de la transmettre à la Commission 20 .

La séance est levée. 